\title{
Article \\ Long-non coding RNAs related to fat deposition in pigs in- cluded lncRNA corresponding to human MALAT1
}

\author{
Katarzyna Piórkowska ${ }^{1}$, Kacper Żukowski ${ }^{2,4^{*}}$, Katarzyna Ropka-Molik ${ }^{1}$ and Mirosław Tyra ${ }^{3}$ \\ 1 National Research Institute of Animal Production, Animal Molecular Biology, Cracow, Poland; \\ katarzyna.piorkowska@iz.edu.pl, katarzyna.ropka@iz.edu.pl \\ 2 National Research Institute of Animal Production, Department of Cattle Breeding, Cracow, Poland; \\ kacper.zukowski@iz.edu.pl \\ 3 National Research Institute of Animal Production, Department of Pig Breeding, Cracow, Poland; \\ miroslaw.tyra@iz.edu.pl \\ 4 Nicolaus Copernicus University, Faculty of Biological and Veterinary Sciences, Torun, Poland \\ * Correspondence: KP: katarzyna.piorkowska@iz.edu.pl; Tel.: +48 666081 316; KZ: \\ kacper.zukowski@iz.edu.pl; Tel.: +48 666081134
}

\begin{abstract}
Obesity is a problem in the last decades since the development of different technologies forced the submission of a faster pace of life, resulting in nutrition style changes. In turn, domestic pigs are an excellent animal model in recognition of adiposity-related processes, corresponding to the size of individual organs, the distribution of body fat in the organism, and similar metabolism. The present study applied the next-generation sequencing method to identify adipose tissue (AT) transcriptomic signals related to increased fat content by identifying differentially expressed genes (DEGs), included long-non coding RNA molecules. The Freiburg RNA tool was applied to recognise predicting hybridisation energy of RNA-RNA interactions. The results indicated several long non-coding RNAs (lncRNAs) whose expression was significantly positively or negatively associated with fat deposition. IncRNAs play an essential role in regulating gene expression by sponging miRNA, binding transcripts, facilitating translation, or coding other smaller RNA regulatory elements. In the pig fat tissue of obese group, increased expression of lncRNAs corresponding to human MALAT1 was observed that previously recognised in the obesity-related context. Moreover, hybridisation energy analyses pinpointed numerous potential interactions between identified differentially expressed lncRNAs, and obesity-related genes and miRNAs expressed in AT.
\end{abstract}

Keywords: lncRNA; obesity; fatness, pig; gene expression regulation; miRNA

\section{Introduction}

Obesity is a problem in the last decades since the development of different technologies forced the submission of a faster pace of life, resulting in diet habit changes. Nowadays, there are used ready-to-eat solutions, fast food restaurants or ready-made lunches offered by various store chains, whose plastic packages often contain harmful substances. Such habitats changes together with increased pesticide food content promotes fat accumulation [1]. Unfortunately, the life rate acceleration is also related to stress, which indirectly favours obesity [2]. The obesity is rapidly spreading civilisation disease entails other severe conditions like cardiovascular disease, diabetes or cancer. Therefore, recognising specific genetic backgrounds that promote or prevent obesity and finding new mechanisms regulating fat deposition seems essential. In numerous studies recognize related-obesity process, domestic pigs are often used as an animal model because their organ size, body fat distribution and metabolism correspond to human [3,4]. 
On the other hand, next-generation sequencing (NGS) successful improved scientific research giving tool to comprehensive analyses of the transcriptome, genome or methylome. The NGS allows, as well, for finding important gene expression regulatory elements included long non-coding RNAs (lncRNAs)[5]. lncRNAs have similar features to mRNA, but they play a regulatory function in gene expression processes, do not code proteins, and are less conserved than mRNA [6]. Long non-coding RNA family includes molecules longer than 200 nucleotides divided into long-intronic ncRNA, intergenic ncRNA (lincRNA), sense lncRNA, anti-sense lncRNA, enhancer-associated lncRNA and circular lncRNA [7]. The function of lncRNA is in the broad sense regulation of gene expression and cell cycle. They can control mechanism of chromatin remodelling [8], they can play a role as 'sponges' for miRNA molecules to relieve or inhibits the binding action of miRNA with target transcripts, which is associated with increased expression of this gene [9]. Finally, some of the lncRNAs expressed from enhancer or silencer regions can bind to target transcripts and enhance [10] or inhibit [11] their subsequent translation.

The role of lncRNAs as epigenetic regulators was also analysed in explaining obesity-related and fat deposition (FD) processes both in humans and animals. Sun et al. [12] identified 1932 lncRNAs expressed in fat tissue and suggested that lnc_000414 is related to fat synthesis by inhibiting the proliferation of intracellular adipocytes. In turn, Yu et al. [13] comparing Duroc and Chinese Luchuan pig backfat pinpointed that differentially expressed lncRNAs are encoded in 13 adipose-related quantitative trait loci. In turn, a recent study by Sun et al. [14] showed that porcine lncRNA - LncIMF4 controls adipogenesis in the intramuscular preadipocytes by relieving autophagy to inhibit lipolysis.

The present study's goal was to identify new potential gene expression regulatory elements, in this case, lncRNAs related to fat deposition and analyse their possible gene interactions. We used pigs as an animal model that belongs to one conservative Polish pig breed, where particular individuals were significantly differed in subcutaneous (SAT) and visceral (VAT) adipose tissue.

\section{Results}

\subsection{Animal Characteristics}

The pig groups used in the experiment significantly differ in fat parameters. The average fat measurement showed that HF pigs had $34 \%$ higher fat content than LF individuals (Figure 1). 


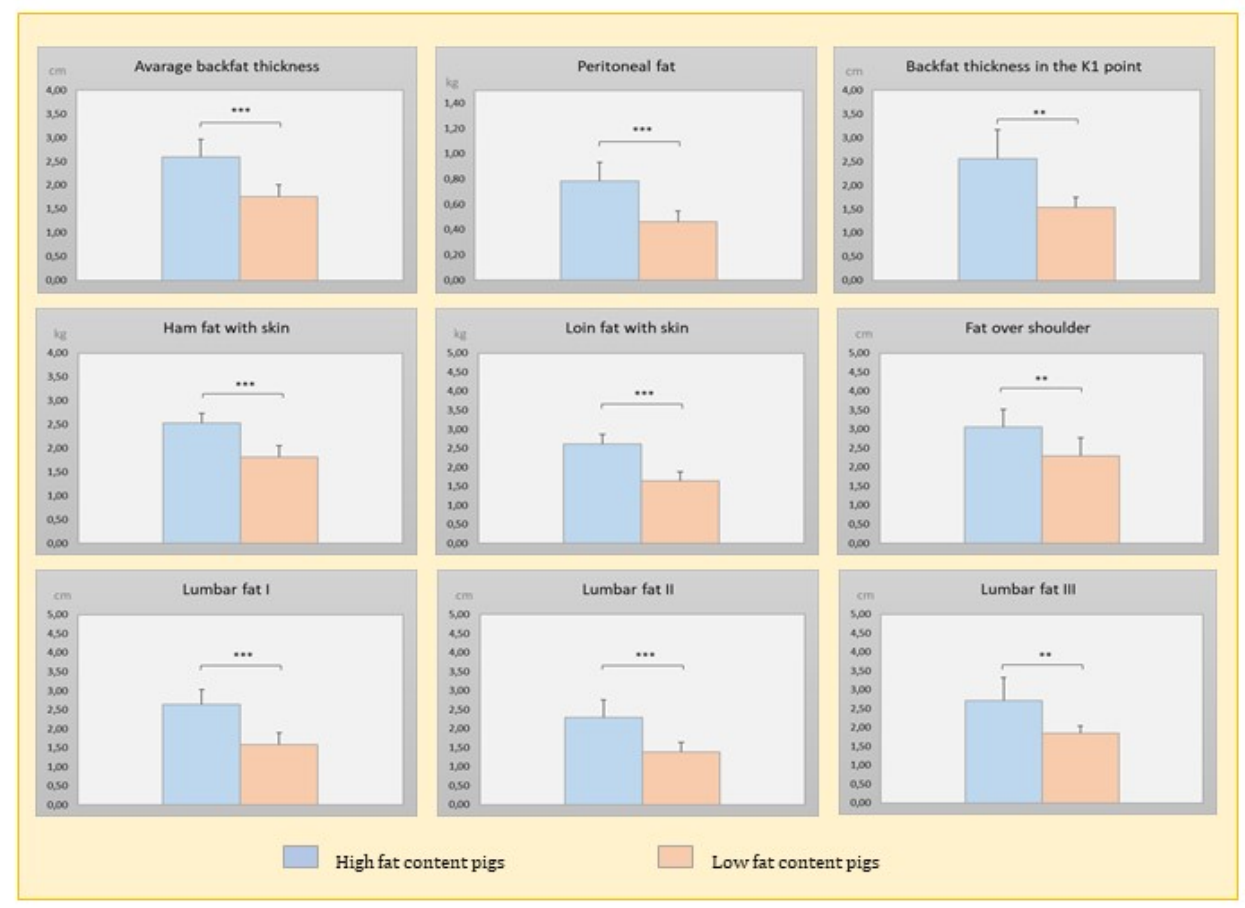

Figure 1. The mean fat trait measurements for low and high FD pigs. K1 point - over the lateral edge of longissimus dorsi muscle.

\subsection{Transcriptome analysis}

The average number of detected row reads per sample was $62,276,008$ and after filtration 61,480,178. After mapping the pig reference genome (Sscrofa11.1 GCA_000003025.6 assembly) (stat compilation - Table S2), 70.8\% uniquely mapped reads of reads matched the annotated exon regions, and $16.5 \%$ matched the introns. One sample was omitted due to a significant low exonic rate $(0.28)$.

The AT transcriptome was analysed across pig groups of different fat content. In the HF group, 103 genes were upregulated that were associated with cholesterol metabolic process (APOB, CES, LEP), inflammatory response (TBXA2R, CCL4, CXCL10, CXCL9, SPP1), response to vitamin (SPP1, CXCL10, CD4, LEP), response to lipid (CD4, CRYAB, TBXA2R, LEP, APOB, CXCL10 and CXCL9), response to hormones (LEP, APOB, SPP1, CD4, CRYAB, TBXA2R, and RHOQ) and toll-like receptor signalling pathway (SPP1, IRF7, CXCL9. CXCL10, CCL4)(Figure 2). Moreover in this fat content group several up-regulated lncRNAs were identified (ENSSSCG00000051330, ENSSSCG00000048856 and ENSSSCG00000048394, ENSSSCG00000047210, ENSSSCG00000047442). In the backfat of LF pigs increased expression of 126 genes was found that were involved in adipocytokine signalling pathway (PCK1), PI3K-Akt signalling pathway (PCK1 and NGFR), thyroxin biosynthesis (DUOX2 and DUOX1) and hormone metabolic process (HSD17B6, CYP1A1, HSD3B1, MME, RBP4, DUOX2, DUOX1, CRABP2), fat cell differentiation (ADIG, GDF10 and NR4A3). Besides, with low fat, three lncRNA (ENSSSCG00000041577, ENSSSCG00000003846, ENSSSCG00000047207) expression was associated. The complete functional analysis results were presented in Table S3. 


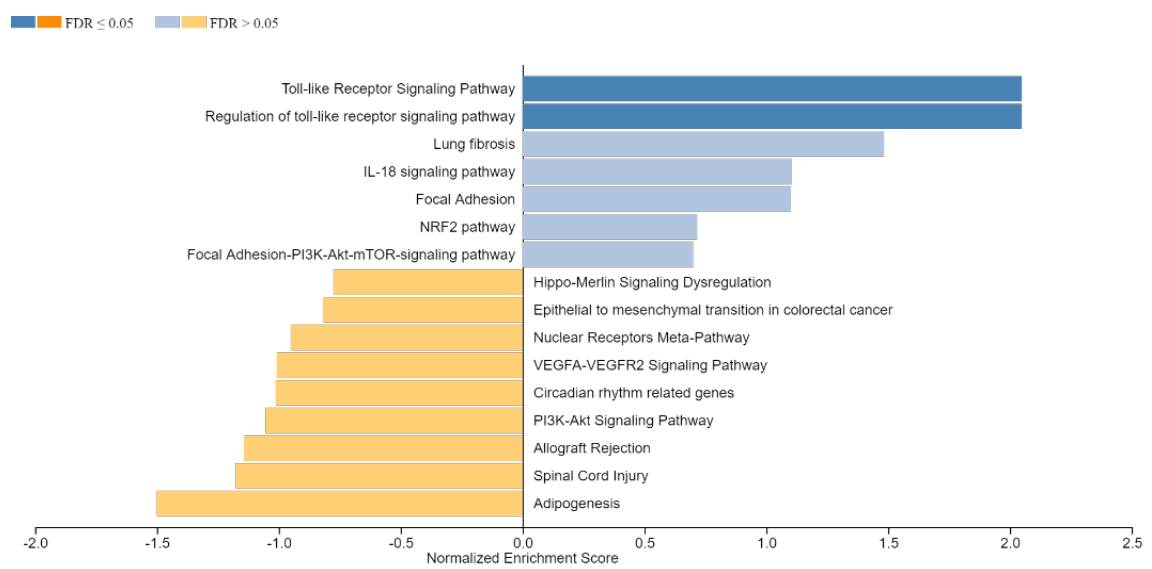

Figure 2. Functional analysis of DEGs identified in fat tissue performed by WebGestalt Gene Set Enrichment Analysis (GSEA).

\subsection{Recognition of IncRNAs correlated with fat deposition}

2.3.1. Positive correlation with fat deposition

1. ENSSSCG00000051330;

ENSSSCG00000051330 is located on chromosome 15:47,623,866-47,625,866 and it highly complements according to NCBI database to HTRA4 gene in sense orientation. Therefore, it is highly probable that this lncRNA is involved in the regulation of HTRA4 expression. Whilst, HTRA4 was found as DEG $(F C=4,04)$ in the present study with positively correlated with FD. However, the Ensemble database shows that ENSSSCG00000051330 is located in the neighbourhood of HTRA4 gene.

2. ENSSSCG00000048394;

This lncRNA contains SINE element PRE-1 P642, which is spread throughout the whole pig genome and is the part of numerous genes related to obesity such as BCL2L13, CD38, CDK7, CHCHD3, CLEC16A, CNR1, DPP8, FLNA, GNAL, GULP1, HIF3A, NOP58, PEX13, RNF216, SERPINE2, SGSM2, TRL10. Therefore, this similarity of sequence, often in the coding regions and anti-sense orientation, ENSSSCG00000048394 can regulate these gene expression. However, none of these genes belong to the DEG group. None of the new isoforms for ENSSSCG00000048394 were detected for this IncRNA.

3. ENSSSCG00000047210;

This lncRNA contains a mobile genetic element (MGE), which is not named yet, nevertheless is spread throughout the whole $\mathrm{S}$. scrofa genome, in genes that are also expressed in fat tissue and are related to obesity ATG4B, CD8A, CLIC6, C1QA, MGAT1, MRPL42, NUDT7, RALGAPB, RNF14, RRAGD, S1PR2, SERPINF1, SPRY1, TIRAP, TNFRSF10A, VTN, WDR41. Therefore, by this similarity of sequence, often in the coding regions and anti-sense orientation, ENSSSCG00000047210 can be involved in regulating these gene expression. However, none of these genes belong to the DEG group. In porcine AT, only one ENSSSCT00000072287 was expressed.

4. ENSSSCG00000047442;

Identified lncRNA positively correlated with FD showed 80\% higher expression in obese pigs than in the lean individuals. Moreover, it shares miscRNA element spread throughout the entire S. scrofa genome with genes, which relationship to obesity was previously described (AACS, ALDH2, ANKRD39, CCDC90B, DAD1, EVL, FATP1, KIFAP3, MED21, NCOA2, OXCT1, PM20D1, RHOBTB3, RYR1, ST3GAL5, ZBTB38, ZNF862). Therefore, by this similarity of sequence, often in the coding regions and anti-sense orientation, ENSSSCG00000047442 can be involved in regulating these gene expression. However, none of these genes belong to the fat-related DEGs. 


\section{ENSSSCG00000048856;}

Blast analysis showed that ENSSSCG00000048856 lncRNA is highly similar to human IncRNA MALAT1. Sanger sequencing and analysis of bam files using Igv2 viewer indicate that porcine MALAT1 has additional isoforms than it was shown in the Ensemble database. New porcine MALAT1 isoform contains exons, intron 1 and 2, as shown in Figure 3, and is the most similar to human isoform ENST00000534336.

\section{A}

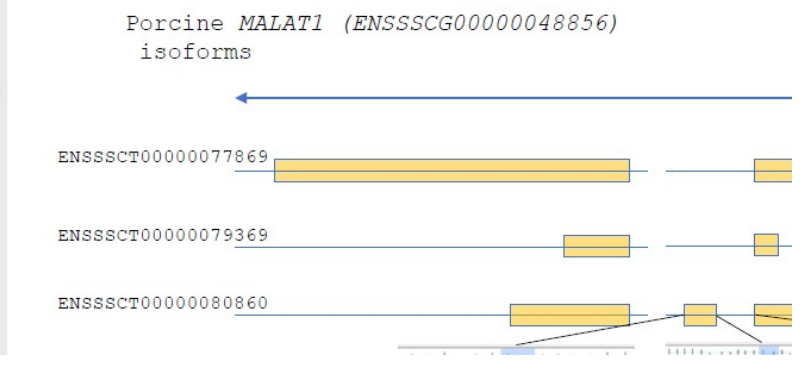

Figure 3. Porcine MALAT1 expression and isoforms identified in fat tissue, after mapping to S.scofa 11.1. The presence of new isoforms was visualised by Ivf2 viewer and confirmed by Sanger sequencing. A. Malatr1 isoforms annotated in Ensemble database. B. MALAT1 visualised by Ivf2 based on identified reads after mapping to S. scrofa genome. C. porcine MALAT1 chromosomal location

2.3.2. Negative correlation with fat deposition

1. ENSSSCG00000003846;

ENSSSCG00000003846 lncRNA, known as GLIS1, showed 2-fold higher expression in lean pigs than in obese. GLIS1 is described as GLIS family zinc finger 1. In humans, it encodes a protein involved in regulating gene expression and functions as activator and repressor of transcription. In pigs, GLIS1 is counted into lncRNA molecules, though it shows high similarity to other GLIS1 orthologues. Nevertheless, in the NCBI database is given PREDICTED: Sus scrofa GLIS family zinc finger 1 (GLIS1), mRNA (XM_021096612) that potential coding Glis1 protein. In porcine AT identified only one (ENSSSCT00000004258) of two GLIS1 isoforms annotated in Ensembl.

2. ENSSSCG00000047207;

ENSSSCG00000047207 was described as LOC106504551 in NCBI database. However, it not showed high similarity to the other lncRNAs in the other species. In the porcine AT one ENSSSCT00000073608 isoform was identified.

3. ENSSSCG00000041577;

In porcine AT four ENSSSCG00000041577 isoforms were detected. This lncRNA shows high similarity to the other ncRNAs such as LOC107034004 (Vicugna pacos), LOC111176067 (Delphinapterus leucas), LOC118002638 (Mirounga leonine), and IncRNA Dio3os (Mus musculus) that is conserved to the opposite strand of deiodinase iodothyronine type III. In turn, Ensembl blast tool shows that ENSSSCG00000041577 pinpoints for MGE (length about $150 \mathrm{bp}$ ) in its sequence that 
is spread throughout pig genome and is the part of EMC7, SUV39H2, MDM4, PABPN1, DNAJC14, UBXN2B, CRNKL1, AQP11, ZNF646, and YPEL5 genes. Besides, ENSSSCG00000041577 seems to be the host for mir1247(NR_031649.1), that it was identified by the NCBI Blast tool.

\subsection{Recognition of $\operatorname{lncRNAs~potential~targets~and~interactions~}$}

Co-expression (threshold $\leq-0.60$ and $\geq 0.60$ ) between raw counts of DE lncRNAs and DEGs using Pearson correlation was estimated. The ENSSSCG00000041577 was co-expressed with the highest DEG number (61\% with negatively and 39\% positively related to FD). I turn, the lowest number of interactions (only 21, with DEGs positively related to FD) were observed for MALAT1 (ENSSSCG00000048856) (Figure S1).
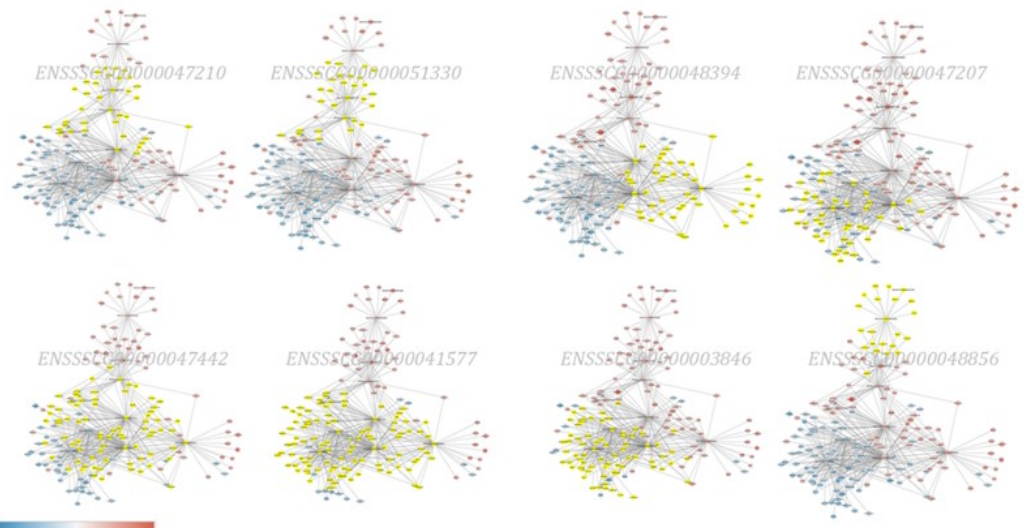

FoldChang

Figure S1. Networks showing the co-expression between DE lncRNAs and DEGs included threshold $\leq-0.60$ and $\geq 0.60$. Yellow nodes present the nearest neighbours. Blue and red saturation described up and down-regulated DEGs; blue down (negatively associated with FD), red up (positively related to fat deposition). The network was generated in Cytoscape 3.8.2.

Analysis of energy hybridisation using the Freiburg IntaRNAv2 tool between DE lncRNAs and DEGs included isoforms expressed in AT showed that ENSSSCG00000047210 lncRNA interacts with the highest of DEG number (62\% were negatively related to FD). In addition, ENSSSCG00000047210 expression was only correlated with positive fat-related DEGs (Figure S2). Moreover, S1 and S2 Figures' comparison showed that MALAT1 and ENSSSCG00000048394 bind much more DEGs based on hybridisation energy assumption than co-expression approach.
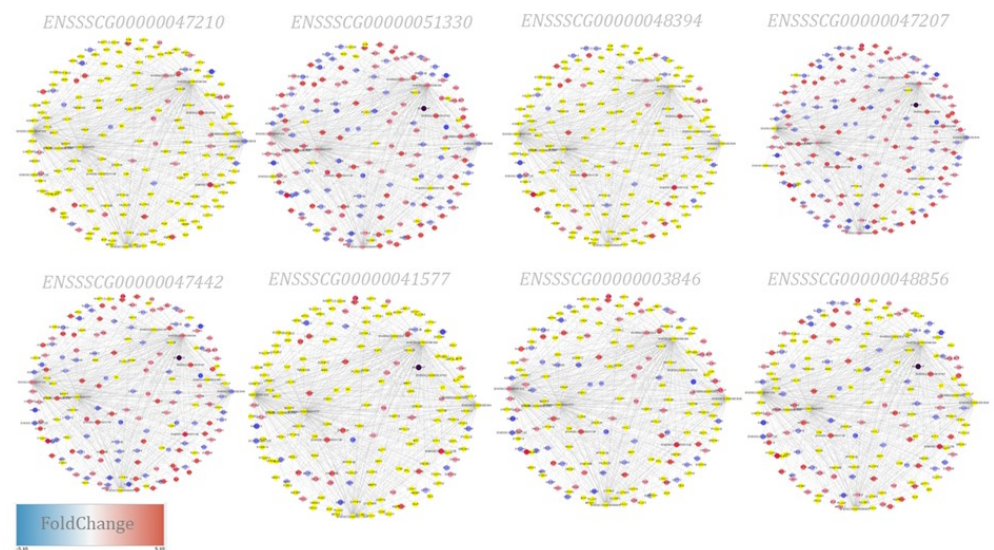
Figure S2. Networks showing the lowest energy between DE lncRNAs and DEGs included energy threshold $\leq-30 \mathrm{kcal} / \mathrm{mol}$. Yellow nodes present the nearest neighbours for particular lncRNAs. Blue and red saturation described up and down-regulated DEGs; blue down (negatively associated with FD, red up (positively related to fat deposition. The network was generated in Cytoscape 3.8.2.

The combined analysis, included both energy and co-expression thresholds, identified closely 180 DE lncRNA-DEGs interactions (Figure 4).

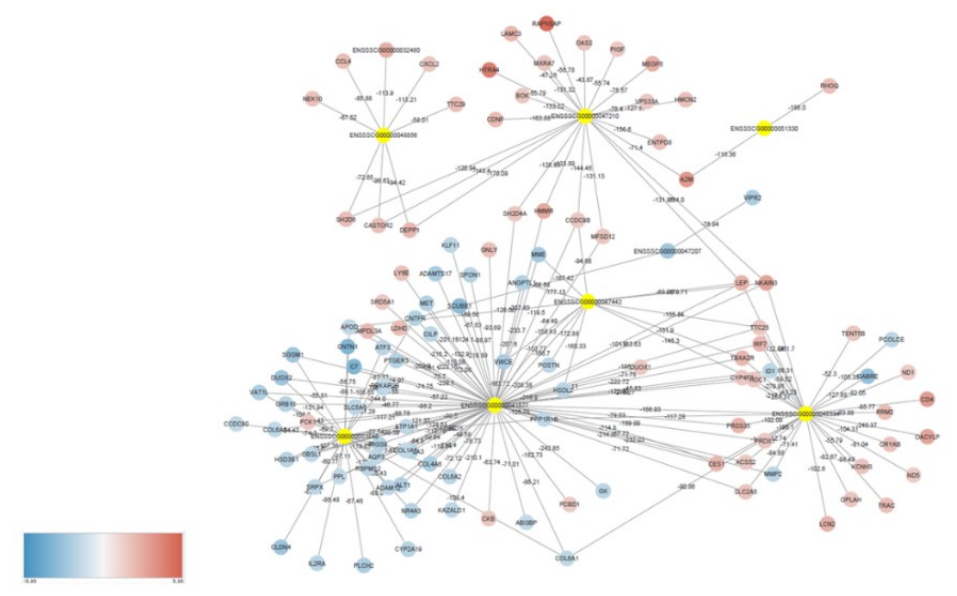

Figure 4. The network presented the theoretical interaction between lncRNA and DEGs based on experimental co-expression analysis (included threshold $\leq-0.60$ and $\geq 0.60$ and data processing using Freiburg IntaRNAv2 tool (Mann et al., 2017) that predict lncRNA-target_mRNA relationship calculating nucleic acid sequence hybridisation energy. Yellow nodes indicate the lncRNAs. Blue and red saturation described up and down-regulated DEGs; blue-down regulated (negatively associated with FD), red-upregulated (positively related to fat deposition. The network was generated in Cytoscape 3.8.2

The functional analysis of interacted DEGs bound by lncRNAs (included co-expression and hybridisation energy approaches) using STRING tool enriched numerous pathways and GO biological processes, including ECM proteoglycan down-regulated DEGs were under control both positive and negative fat-related lncRNAs. The next observation after function analysis pinpointed that LEP involved in regulating energy balance, showing a 3.5-fold change, can be even under the control of four DElncRNAs because their theoretical hybridisation energy was low. LEP enriched GO biological processes such as glucose and lipid metabolic, immune response, response to nutrient, and hormone metabolic (Figure 5). ENSSSCG00000041577 interacted with DEGs that enriched ECM proteoglycan, lipid and glucose metabolism processes. The complete results of co-expression, the Freiburg IntaRNAv2 between lncRNA, DEGs and miRNA analyses were presented in Table S4. 

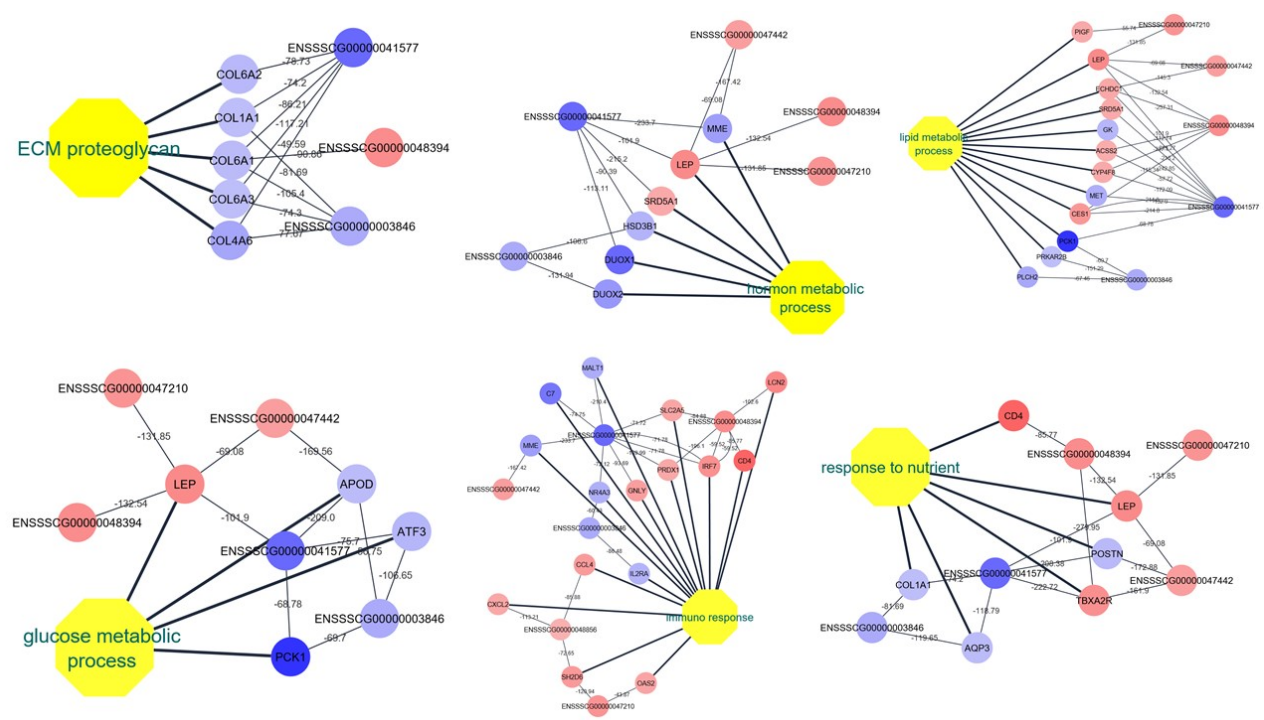

Figure 5. The networks show the enriched pathways and GO metabolic process analysed using STRING tool and generated by Cytoscape 3.8.2 for DEGs interacted with lncRNA included co-expression threshold $\leq-0.60$ and $\geq 0.60$, and energy $\leq-30 \mathrm{kcal} / \mathrm{mol}$. On edges are presented hybridisation energy calculated based on nucleic acid sequence features. Yellow node indicates enriched pathway/GObiological process. Blue and red saturation describe up-and down-regulated DEGs. Blue - downregulated (negatively associated with FD), red - upregulated (positively related to FD).

\subsection{Validation of RNA-seq results}

The validation of RNAseq results was performed using qPCR method and Pearson correlation. The qPCR confirmed RNAseq outcomes. However, ENSSSCG00000003846 and ENSSSCG00000051330 R coefficients were low that could be associated with gene complexity, numerous polymorphic variants and slightly changes in isoforms in comparison to Ensemble data. The FoldChange and R coefficient were presented in Figure 6.

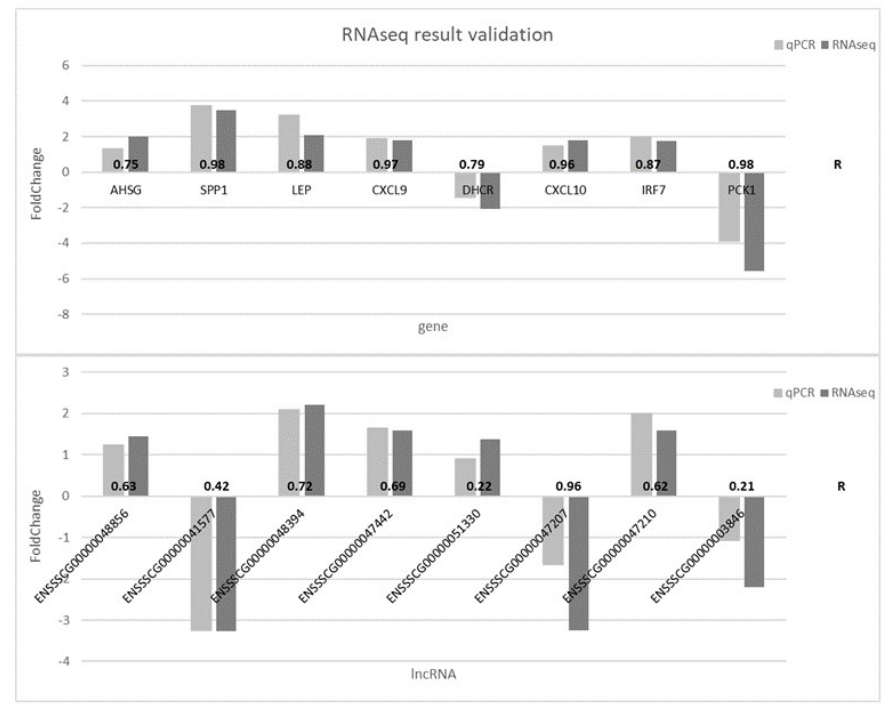

Figure 6. Validation of RNAseq results. On plots, fold change values were presented. R- correlation coefficient .

\section{Discussion}


lncRNAs play an essential role in the regulation of gene expression, as was mentioned before, they can regulate this process at different molecular level as anti-sense transcripts [24], miRNA catchers [25] or translation inhibitors [26]. In the present study, we would like to find novel lncRNAs related to the regulation of FD in pigs, which information can also be crucial for researchers to struggle with the problem of obesity in humans.

The present differentially expressed gene analysis using DESeq2 tool showed that eight lncRNAs were differentially expressed, five positively and three negatively related to FD. Among the first group was lncRNA ENSSSCG00000048856, which was highly similar to the MALAT1 gene described in many species, including humans. MALAT1 called Metastasis Associated Lung Adenocarcinoma Transcript 1 [27] initially was identified as a prognostic marker of poor outcomes in patients with early-stage non-small cell lung cancer [28]. It is the most abundant of lncRNA molecules in mouse cortex and liver [29]. It regulates pre-mRNA [30] because it binds to pre-mRNA splicing factors in the nuclear speckles and regulates their distribution and splicing activity [31]. Nevertheless, observation in knockout MALAT1-/- mice did not indicate any splicing disturbance, and the authors found that Malat1 transcription is dispensable for mouse pre-and post-natal development [32]. However, Malat1 gene inactivation results in a nearly two-fold up-regulation of several genes that reside adjacent to its locus, which suggested the important role of the MALAT1 promoter. In turn, its role in the metastasis was well supported by numerous reports [33,34]. The next study reported that MALAT1 expressed in cardiomyocytes is induced by saturated fatty acid during palmitic acid-induced hepatic steatosis treatment. And its downregulation alleviated SFA-induced myocardial inflammatory injury via miR-26a/HMGB1/TLR4/NF- $\kappa B$ axis [35]. However, our Freiburg IntaRNAv2 analysis did not indicate that porcine MALAT1 bind miR-26a because their hybridisation energy was over $-15 \mathrm{kcal} / \mathrm{mol}$. MALAT1 was also considered previously in the obesity-relation context. Also, the present study found increased MALAT1 expression in obese ZW gilts. Earlier report Yan et al. [36] suggested that MALAT1 promotes hepatic steatosis by increased stability of SREBP-1c protein. Liu et al. [37] presented that it is involved in regulating cholesterol accumulation in ox-LDL-induced macrophages via microRNA-17-5p/ABCA1 axis. The study analysed MALAT1, and TUG1 expression in AT in the obesity context showed no differences between obese and normal women, but MALAT1 mRNA level in SAT was positively correlated with SREBP-1c, PPAR $\gamma$, and their targets; FAS and ACACA, as well as with VAT mRNA levels of PGC1 $\alpha$ [38]. In the present study, SREBP-1c, PPAR $\gamma$, FAS and ACACA, were not DE, but an extra co-expression analysis showed that the MALAT1 mRNA level in SAT was positively correlated FAS and ACACA. On the other hand, the present Freiburg IntaRNAv2 analysis predicted that porcine MALAT1 could interact with numerous identified DEGs that enriched the lipid response (CRYAB, RGS9, SRD5A1, LEP, CNP. NGFR, NR4A3, ATP1A1, CXCL9, CXCL2, CD4, TBXA2R, COL1A1). However, their hybridisation energy was not so low and oscillated in the range of -45 to $-30 \mathrm{kcal} / \mathrm{mol}$. Nevertheless, our observation suggests the possible LEP-MALAT1 interaction that was proposed before when decreased MALAT1 expression in SAT of ob/ob (leptin deficiency), db/db (leptin receptor deficiency) mice models and in diet-induced obesity mice was observed [39]. Nevertheless, KO MALAT1 mice gained as much weight and developed insulin resistance to a similar extent as their Malat1+/+ littermates [39]. On the other hand, the present Freiburg IntaRNAv2 test showed for possible sponging of a few miRNAs by MALAT1, included ssc-miR-125a-5p. This miRNA promotes the proliferation and inhibition of differentiation of 3T3-L1 preadipocyte by binding to STAT3 [40]. Therefore, MALAT1 bound ssc-miR-125a-5p could reduce preadipocyte number and in the consequence adipocytes, and could be the attempt of limited excessive fat content. In the present study, MALAT1 was not co-expressed with numerous DEGs but analysis of hybridisation energy showed that it can regulate even 85 of them. It suggests that MALAT1 can act at various molecular level not only transcript blocking[41]. Thus, our observa- 
tion and previous literature reports persuade us to continue researching MALAT1 in the obesity regulation context because the interactions are still not clear.

Besides ENSSSCG00000048856 lncRNA - porcine MALAT1, ENSSSCG00000003846 GLIS1 found its orthologues in the other species and its molecular functions were investigated before. For example, cattle study supported its role in embryo development[42]. Scoville et al. [43] reported GLIS1 function in the promotion of reprogramming human and mice fibroblasts into an induced pluripotent stem cell (iPSCs). In turn, some human GLIS1 variants increased neurological disease risk for diseases such as Alzheimer [44] or late-onset Parkinson [45]. The recently Tosic et al.[46] study presented a promoting role of GLIS1 in brown adipocyte (BAT) differentiation and described it as a novel pro-adipogenic transcription factor. The authors observed that GLIS1 in mouse myoblast C2C12 cells decreased myogenic markers' expression and enhanced adipogenic marker expression. However, in pigs previously reports showed the lack of brown adipocytes [47]. Moreover, Joshi et al.[48] established by adipocytes' expression profile in the obese patient that expression of numerous obesity-related genes was under GLIS1 transcription factor control. In the present study, GLIS1 transcript level was negatively associated with fat deposition ( $\mathrm{FC}=2$ between experimental groups). The presented GLIS1 expression profile is valuable preliminary research, but limited information about its function in the adipose tissue thus the need for continuing GLIS1 study in the obesity context.

Unfortunately, the other identified in the present study DE lncRNAs do not find the orthologues, enabling us to understand their functions. However, the current statistical analysis identified that ENSSSCG00000041577, ENSSSCG00000047210 and ENSSSCG00000048394 were co-expressed with numerous DEGs and Freiburg IntaRNAv2 analysis pinpointed for low hybridisation energy of their interactions. Among these target genes was LEP (mentioned before), which protein is secreted by fat tissue and it plays a crucial role in regulating feed intake and fat deposition [49,50]. Moreover, significant low energy was identified for APOD gene interactions. APOD encodes apolipoprotein $\mathrm{D}$ and in the present study was found that its expression is negatively related to fat deposition ( $\mathrm{FC}=1.54)$. Apolipoproteins are ingredients of lipoproteins that play a crucial role in lipid homeostasis. ApoD is critical to triglyceride metabolism. Its circulating form is present in high-density lipoprotein (HDL), and in $\mathrm{db} / \mathrm{db}$ mice with visceral obesity and altered lipid metabolism, the reduced level in plasma was observed [51]. Besides, obese mice with increased apoD showed improved triglyceride (TG) profiles. ApoD also promotes LPL-mediated hydrolysis of VLDL in vitro, which correlated with its TG-lowering action in vivo. In turn, genetic variants of apoD are associated with developing metabolic syndrome. The present study also observed that ENSSSCG00000041577 lncRNA expression is positively (0.70) and ENSSSCG00000048394 negatively (-0.52) correlated with APOD mRNA level that can suggest possible transcript regulation.

On the other hand, interaction miRNA-lncRNA analysis indicated that ENSSSCG00000047210, ENSSSCG00000003846 (GLIS1), and ENSSSCG00000048394 show low hybridisation energy for interactions with ssc-mir-709. This microRNA was described previously as anti-adipogenic miRNAs [21] because it inhibits 3T3-L1 cell differentiation by targeting GSK3 $\beta$ belonged to Wnt/ $\beta$-catenin signalling [52]. However, Ropka-Molik et al. (2020) did not found the relationship between this miRNA and fat deposition investigating different pig breeds raised in Poland.

It is challenging to know the molecular function of lncRNAs with no equivalent in the other species without experimental analysis using, for example, cell culture. Our genetic database searching indicated that four of DElncRNAs contain MGEs, that in the lncRNAs are termed as Repeat Insertion Domains of LncRNAs (RIDLs)[53]. These elements, called transposable element-derived (TE-derived) fragment of lncRNA can act as RNA-, DNA-, and protein-binding domains. Therefore, RIDLs can have a regulatory role in gene expression. The use of NCBI and Ensembl blast tools and RNAcental database enabled finding the same MGEs in the other genes expressed in fat tissue related to obe- 
sity and fat deposition. In ENSSSCG00000048394 lncRNA is located PRE-1 P642 mobile element, which is also spread in the HIF3A encoding hypoxia-inducible factor $3 \mathrm{a}$, as well as, in the TLR10 and RNF216 encoding Toll-Like Receptor 10 and Ring Finger Protein 216. HIF3A expression was upregulated in AT in obese and type 2 diabetes (T2D) patients correlating with BMI [54]. Increased expression of RNF216 was found in peripheral blood mononuclear cells of subjects, which regain more weight after a successful initial weight loss [55]. In ENSSSCG00000047210 lncRNA and RALGAPB gene also included the same mobile genetic element. RALGAPB encodes Ral GTPase Activating Protein Non-Catalytic Subunit Beta and seems to be involved in fat deposition regulation. Such function was confirmed on mice model with RalGAPB knockout used diet-induced obesity were protected against metabolic disease via better glucose uptake by BAT. In turn, small G protein RalA activated by RalGAPB is believed that play a crucial role in glucose transport in AT in vivo [56]. The present study confirmed that MGE was also found in ENSSSCG00000041577 lncRNA, which shares it with other numerous obesity-related genes.

At the end of our lncRNA consideration, it was found that ENSSSCG00000041577 is the host for mir1247. Few previously reports indicate the role of this microRNA in the metabolic regulation process in AT. For example, mice with a high-fat diet exhibited in the epididymal AT significant downregulation of miR-1247 compared to mice with regular diet [57]. And more, the miR-1247 was upregulated in the liver of obesity-resistant mice compared to prone-obesity subjects raised with high-fat diet [58]. Therefore, the ENSSSCG00000041577 can be the source of miR-1247 that plays a significant role in the counteraction of obesity effects.

\section{Materials and Methods}

\subsection{Animals}

The study was conducted on 16 unrelated gilts of the Złotnicka White (ZW) breed presenting high $(\mathrm{HF}, \mathrm{n}=8)$ and low $(\mathrm{LF}, \mathrm{n}=8)$ level of fat deposition. $\mathrm{ZW}$ belonging to native Polish pig breed was included in the National Preservation Program. Thus they were not under artificial selection pressure. Animals were maintained at the Pig Test Station in Chorzelów under the same environmental and feeding conditions. The animals were delivered to pig test station as piglets, and they were fed ad libitum from 30 up to $100( \pm 2.5)$ $\mathrm{kg}$ of weight, then were slaughtered and dissected. The fat content parameters were measured during dissection according to Pig Test Station procedure. The level of fat content measurements was estimated based on a few fat parameters such as loin backfat mass, subcutaneous fat mass, ham fat mass, knuckle fat mass, backfat thickness by a shoulder, backfat thickness by ridge and three other measurements of fat content. Up to 20 minutes after dissection backfat tissue samples at the last rib level were collected and stabilised by RNAlater solution and stored in $-20{ }^{\circ} \mathrm{C}$.

\subsection{NGS library construction}

RNA was isolated using Synegen kit according to the manufacturer's protocol. Tissue samples with beads using a Bullet Blender 24 homogeniser (Next Advance) were homogenised. The RNA (Agencourt RNAClean XP kit, Backman) was purified by a bead method, and its quality and quantity were assessed by a Qubit Fluorometer (Invitrogen) and by a TapeStation 2200 system (RNA tapes, Agilent). RIN parameters were in the range 7,5-8,5. cDNA libraries were prepared using TruSeq RNA Sample Preparation Kit v2 (Illumina) from $300 \mathrm{ng}$ aliquots of purified RNA samples according to the manufacturer's protocol. Then, the libraries were quantity using the Qubit 2.0 Fluorometer and 2200 TapeStation (with D1000 screen tapes). The cDNA libraries' final concentration was normalised to $10 \mathrm{nM}$, after which the libraries were pooled. Transcriptome sequencing was performed in 150PE cycles on HiSeq 3000 System (Illumina, San Diego, CA, USA) (Admera Health Biopharma Services commissioned sequencing). 


\subsection{Aligning raw reads to the pig transcriptome}

The raw sequences were qualitatively controlled using the FastQC tool. Flexbar software was used to remove Illumina adapters and poly-A sequences and reads shorter than 36 bp or with a quality score lower than the default of 20 from the dataset were removed. Filtered sequences to the Sus scrofa genome (Sscrofa11.1.100 assembly) were aligned with reference annotation containing the 31908 genes and 63041 transcripts annotated in the Ensembl database. Alignment and estimation of the counts level were assessed using the RSEM package supported by STAR aligner. The alignment and DEG statistics were generated using the Picard tools (https://broadinstitute.github.io/picard/) and RNA-SeQC tools (https://software.broadinstitute.org/cancer/cga/rna-seqc). The sequence data has been submitted to the Gene Expression Omnibus (accession no. GSE160436).

\subsection{The DEGs analysis}

The DEGs, isoforms, and corresponding P-values were estimated using DESeq2 [15] statistical methods. All P-values were adjusted to control for false discovery rates due to the multiple testing procedures used. To control for type I errors, the adjusted P-value was set at $<0.05$. The level of statistical significance for DEGs between two pig's groups.

\subsection{Recognation of DE IncRNAs and calculation potential interactions with their targets}

DE lncRNAs were recognised using RNAcentral and Blast in NCBI and Ensemble tools. The Freiburg IntaRNAv2 tool [16] was used to predict lncRNA-miRNA and lncRNA-target mRNA interaction based on the nucleic acid sequence. For lncRNA-miRNA used default parameters: minimum seven basepairs in seed, no lonely base pairs, no GU at helix ends, ignore seeds with GU ends, maximum interaction energy $-15 \mathrm{kcal} / \mathrm{mol}$, and hybridisation energy $-16.5 \mathrm{kcal} / \mathrm{mol}$. As input miRNA data, the DE miRNA sequences (included isomiRs) identified in our previous study (GSE122816) was used [17] and fat-related miRNA sequences mentioned in the literature [18-21]. For lncRNA-target_mRNA interaction analysis used default parameters: minimum ten basepairs in seed, no lonely base pairs, no GU at helix ends, ignore seeds with GU ends, and a threshold was set at maximum interaction energy $-30 \mathrm{kcal} / \mathrm{mol}$ and hybridisation energy $-35 \mathrm{kcal} / \mathrm{mol}$. As potential targets, the identified in the present study DEGs were tested, including particular isoforms expressed in fat tissue. The functional analysis for potential lncRNA targets was performed using PANTHER 16.0 (S.scrofa reference), WebGestalt Gene Set Enrichment Analysis (GSEA) and STRING v.11 (H.sapience reference) tools, included p-value adjusted due to multiple analysis. In turn, Cytoscape visualised interaction between lncRNAs and their targets.

\section{6. qPCR analysis and identification DE $\operatorname{lncRNA}$ isoforms}

qPCR analysis was used to validate the RNAseq results. Eight genes encoding proteins (AHSG, SPP1, LEP, CXCL9, CXCL10, DHCR24, IRF7, and PCK1) and all identified DE lncRNAs were used to validate RNA-seq results.

Analysis of gene expression was performed included all ZW pig used in RNAseq method. As an endogenous control, the three genes OAZ1, RPS29 and RPL27 were used; they are stable regarding the expression between various breeds and tissues [22]. qPCR analysis was performed using High sensitivity EvaGreen mix and High sensitivity Prope mix (A\&A biotechnology) and primers designed in Primer3 and assays purchased by Applied Biosystem (Table S1). The identification of isoforms for particular lncRNAs, bam files (RNA-seq data) and Sanger sequencing method was applied. Sanger sequencing was performed on cDNA additional purified by Agencourt RNAClean XP kit, Backman to remove all DNA contamination. 


\subsection{Statistical calculation}

The relative quantity of transcript after qPCR was calculated by ddCT method [23]. The significant correlation index was calculated based on Pearson correlation (SAS Enterprise v. 7.1). Correlation analysis of DE lncRNAs and DEGs was performed using raw counts obtained after mapping to Sus scrofa 11.1 genome assembly. The threshold was estimated at 0.60 correlation factor with $\mathrm{P}<0.05$. Co-expression analysis was calculated based on the Pearson correlation (Sas Enterprise v. 7.1).

\section{Conclusions}

The present study showed a possible involvement of a few lncRNAs in fat deposition regulation and playing a role in fat metabolism processes. Moreover, there were presented possible approaches to recognise lncRNA function, wide act spectrum molecules, in which the abilities are unknown. Moreover, found increased expression of MALAT1 in obese subjects, lncRNA which role in obesity is still considered, often questioned or doubtful. Overall, the present study that used pigs as an animal model expands current knowledge on possible gene expression regulation by lncRNAs in fat tissue. And our future study will engage the 3T3-L1 preadipocyte line in the experimental analysis of lncRNA function.

\section{Supplementary Materials:}

Figure S1. Networks showing the co-expression between DE lncRNAs and DEGs included threshold $\leq-0.60$ and $\geq 0.60$. Yellow nodes present the nearest neighbours. Blue and red saturation described up and down-regulated DEGs; blue down (negatively associated with FD), red up (positively related to fat deposition). The network was generated in Cytoscape 3.8.2.

Figure S2. Networks showing the lowest energy between DE lncRNAs and DEGs included energy threshold $\leq-30 \mathrm{kcal} / \mathrm{mol}$. Yellow nodes present the nearest neighbours for particular lncRNAs. Blue and red saturation described up and down-regulated DEGs; blue down (negatively associated with FD, red up (positively related to fat deposition. The network was generated in Cytoscape 3.8.2.

Table S1. qPCR analysis and identification DE lncRNA isoforms.

Table S2. The statistics compilation (preprocessing, alignment, RNASeq QC).

Table S3. The functional analysis.

Author Contributions: Conceptualization, K.P. 1; Investigation, K.P. 1, M.T., K R-M Methodology, K.P., MT Resources, Validation, K.P.1; Visualization, K.P.1; K.Ż.; Software, K.Ż., Project administration, K.P.., Writing - original draft preparation, K.P.1, Writing - review and editing, K.P.1, M.T. K.Ż. K R.-M. Supervision, K.P. M.T.; Funding acquisition, K.P.; K.Ż.; M.T.

Funding: This study was supported by statutory activity of National Research Institute of Animal Production no. 01-18-05-21 and BIOSTRATEG2/297267/14/NCBR/2016.

Ethical approval: The research has been performed on biological material derived from pigs maintained and slaughtered in the Test Pig Station (National Research Institute of Animal Production). In the Test Station pigs were slaughtered, dissected and after carcass evaluation, meat was standard intended for consumption. Therefore, our research does not require the approval of Animal Experimentation committee.

Conflicts of Interest: None of the authors has a financial or other relationship with other people or organizations that may inappropriately influence this work.

\section{References}

1. Carvalho, F.P. Pesticides, environment, and food safety. Food Energy Secur. 2017, 6, 48-60.

2. Scott, K.A.; Melhorn, S.J.; Sakai, R.R. Effects of Chronic Social Stress on Obesity. Curr. Obes. Rep. 2012, 1, 16-25, doi:10.1007/s13679-011-0006-3. 
3. Stachowiak, M.; Szczerbal, I.; Switonski, M. Genetics of Adiposity in Large Animal Models for Human Obesity-Studies on Pigs and Dogs. In Progress in Molecular Biology and Translational Science; Elsevier B.V., 2016; Vol. 140, pp. 233-270 ISBN 9780128046159 .

4. Nielsen, K.L.; Hartvigsen, M.L.; Hedemann, M.S.; Lræke, H.N.; Hermansen, K.; Bach Knudsen, K.E. Similar metabolic responses in pigs and humans to breads with different contents and compositions of dietary fibers: A metabolomics study. Am. J. Clin. Nutr. 2014, 99, 941-949, doi:10.3945/ajcn.113.074724.

5. Sun, L.; Lin, J.D. Function and mechanism of long noncoding RNAs in adipocyte biology. Diabetes 2019, 68, 887-896, doi:10.2337/dbi18-0009.

6. Diederichs, S. The four dimensions of noncoding RNA conservation. Trends Genet. 2014, 30, 121-123, doi:10.1016/j.tig.2014.01.004.

7. St.Laurent, G.; Wahlestedt, C.; Kapranov, P. The Landscape of long noncoding RNA classification. Trends Genet. 2015, 31, 239-251, doi:10.1016/j.tig.2015.03.007.

8. Böhmdorfer, G.; Wierzbicki, A.T. Control of Chromatin Structure by Long Noncoding RNA. Trends Cell Biol. 2015, 25, 623-632, doi:10.1016/j.tcb.2015.07.002.

9. Cesana, M.; Cacchiarelli, D.; Legnini, I.; Santini, T.; Sthandier, O.; Chinappi, M.; Tramontano, A.; Bozzoni, I. A long noncoding RNA controls muscle differentiation by functioning as a competing endogenous RNA. Cell 2011, 147, 358-369, doi:10.1016/j.cell.2011.09.028.

10. Ørom, U.A.; Derrien, T.; Beringer, M.; Gumireddy, K.; Gardini, A.; Bussotti, G.; Lai, F.; Zytnicki, M.; Notredame, C.; Huang, Q.; et al. Long noncoding RNAs with enhancer like function in human cells. Cell 2010, 143, 46-58, doi:10.1016/j.cell.2010.09.001.

11. Kino, T.; Hurt, D.E.; Ichijo, T.; Nader, N.; Chrousos, G.P. Noncoding RNA Gas5 is a growth arrest- and starvation-associated repressor of the glucocorticoid receptor. Sci. Signal. 2010, 3, doi:10.1126/scisignal.2000568.

12. Sun, Y.; Chen, X.; Qin, J.; Liu, S.; Zhao, R.; Yu, T.; Chu, G.; Yang, G.; Pang, W. Comparative Analysis of Long Noncoding RNAs Expressed during Intramuscular Adipocytes Adipogenesis in Fat-Type and Lean-Type Pigs. J. Agric. Food Chem. 2018, 66, 12122-12130, doi:10.1021/acs.jafc.8b04243.

13. Yu, L.; Tai, L.; Zhang, L.; Chu, Y.; Li, Y.; Zhou, L. Comparative analyses of long non-coding RNA in lean and obese pig. Oncotarget 2017, 8, 41440-41450, doi:10.18632/oncotarget.18269.

14. Sun, Y.; Cai, R.; Wang, Y.; Zhao, R.; Qin, J.; Pang, W. A newly identified LNcRNA LncIMF4 controls adipogenesis of porcine intramuscular preadipocyte through attenuating autophagy to inhibit lipolysis. Animals 2020, 10, doi:10.3390/ani10060926.

15. Love, M.I.; Huber, W.; Anders, S. Moderated estimation of fold change and dispersion for RNA-seq data with DESeq2. Genome Biol. 2014, 15, doi:10.1186/s13059-014-0550-8.

16. Mann, M.; Wright, P.R.; Backofen, R. IntaRNA 2.0: Enhanced and customizable prediction of RNA-RNA interactions. Nucleic Acids Res. 2017, 45, W435-W439, doi:10.1093/nar/gkx279.

17. Ropka-Molik, K.; Pawlina-Tyszko, K.; Żukowski, K.; Tyra, M.; Derebecka, N.; Wesoły, J.; Szmatoła, T.; Piórkowska, K. Identification of Molecular Mechanisms Related to Pig Fatness at the Transcriptome and miRNAome Levels. Genes (Basel). 2020, 11, 600, doi:10.3390/genes11060600.

18. Zhu, Y.-L.; Chen, T.; Xiong, J.-L.; Wu, D.; Xi, Q.-Y.; Luo, J.-Y.; Sun, J.-J.; Zhang, Y.-L. miR-146b Inhibits Glucose Consumption by Targeting IRS1 Gene in Porcine Primary Adipocytes. Int. J. Mol. Sci. 2018, 19, 783, doi:10.3390/ijms19030783.

19. Song, Z.; Cooper, D.K.C.; Cai, Z.; Mou, L. Expression and regulation profile of mature microRNA in the pig: Relevance to xenotransplantation. Biomed Res. Int. 2018, 2018, doi:10.1155/2018/2983908.

20. Du, J.; Xu, Y.; Zhang, P.; Zhao, X.; Gan, M.; Li, Q.; Ma, J.; Tang, G.; Jiang, Y.; Wang, J.; et al. MicroRNA-125a-5p Affects Adipocytes Proliferation, Differentiation and Fatty Acid Composition of Porcine Intramuscular Fat. Int. J. Mol. Sci. 2018, 19, 501, doi:10.3390/ijms19020501.

21. Iacomino, G.; Siani, A. Role of microRNAs in obesity and obesity-related diseases. Genes Nutr. 2017, 12, doi:10.1186/s12263-017-0577-z.

22. Piórkowska, K.; Ropka-Molik, K.; Eckert, R.; Rózycki, M. The expression pattern of proteolytic enzymes of cathepsin family in two important porcine skeletal muscles. Livest. Sci. 2013, 157, 427-434, doi:10.1016/j.livsci.2013.09.002.

23. Pfaffl, M.W.; Tichopad, A.; Prgomet, C.; Neuvians, T.P. Determination of stable housekeeping genes, differentially regulated target genes and sample integrity: BestKeeper - Excel-based tool using pair-wise correlations. Biotechnol. Lett. 2004, 26, 509-515, doi:10.1023/B:BILE.0000019559.84305.47.

24. Liu, X.; Li, D.; Zhang, D.; Yin, D.; Zhao, Y.; Ji, C.; Zhao, X.; Li, X.; He, Q.; Chen, R.; et al. A novel antisense long noncoding RNA, TWISTED LEAF, maintains leaf blade flattening by regulating its associated sense R2R3-MYB gene in rice. New Phytol. 2018, 218, 774-788, doi:10.1111/nph.15023.

25. Zhang, X.; Zhou, Y.; Chen, S.; Li, W.; Chen, W.; Gu, W. LncRNA MACC1-AS1 sponges multiple miRNAs and RNA-binding protein PTBP1. Oncogenesis 2019, 8, 1-13, doi:10.1038/s41389-019-0182-7.

26. Zhang, X.; Wang, W.; Zhu, W.; Dong, J.; Cheng, Y.; Yin, Z.; Shen, F. Mechanisms and functions of long non-coding RNAs at multiple regulatory levels. Int. J. Mol. Sci. 2019, 20, doi:10.3390/ijms20225573.

27. Amodio, N.; Raimondi, L.; Juli, G.; Stamato, M.A.; Caracciolo, D.; Tagliaferri, P.; Tassone, P. MALAT1: A druggable long non-coding RNA for targeted anti-cancer approaches. J. Hematol. Oncol. 2018, 11, 1-19, doi:10.1186/s13045-018-0606-4. 
28. Ji, P.; Diederichs, S.; Wang, W.; Böing, S.; Metzger, R.; Schneider, P.M.; Tidow, N.; Brandt, B.; Buerger, H.; Bulk, E.; et al. MALAT-1, a novel noncoding RNA, and thymosin $\beta 4$ predict metastasis and survival in early-stage non-small cell lung cancer. Oncogene 2003, 22, 8031-8041, doi:10.1038/sj.onc.1206928.

29. Zhang, B.; Arun, G.; Mao, Y.S.; Lazar, Z.; Hung, G.; Bhattacharjee, G.; Xiao, X.; Booth, C.J.; Wu, J.; Zhang, C.; et al. The lncRNA malat1 is dispensable for mouse development but its transcription plays a cis-regulatory role in the adult. Cell Rep. 2012, 2, 111-123, doi:10.1016/j.celrep.2012.06.003.

30. Tripathi, V.; Ellis, J.D.; Shen, Z.; Song, D.Y.; Pan, Q.; Watt, A.T.; Freier, S.M.; Bennett, C.F.; Sharma, A.; Bubulya, P.A.; et al. The nuclear-retained noncoding RNA MALAT1 regulates alternative splicing by modulating SR splicing factor phosphorylation. Mol. Cell 2010, 39, 925-938, doi:10.1016/j.molcel.2010.08.011.

31. Singh, D.K.; Prasanth, K. V. Functional insights into the role of nuclear-retained long noncoding RNAs in gene expression control in mammalian cells. Chromosom. Res. 2013, 21, 695-711, doi:10.1007/s10577-013-9391-7.

32. Eißmann, M.; Gutschner, T.; Hämmerle, M.; Günther, S.; Caudron-Herger, M.; Groß, M.; Schirmacher, P.; Rippe, K.; Braun, T.; Zörnig, M.; et al. Loss of the abundant nuclear non-coding RNA MALAT1 is compatible with life and development. RNA Biol. 2012, 9, 1076-1087, doi:10.4161/rna.21089.

33. Kim, J.; Piao, H.L.; Kim, B.J.; Yao, F.; Han, Z.; Wang, Y.; Xiao, Z.; Siverly, A.N.; Lawhon, S.E.; Ton, B.N.; et al. Long noncoding RNA MALAT1 suppresses breast cancer metastasis. Nat. Genet. 2018, 50, 1705-1715, doi:10.1038/s41588-018-0252-3.

34. Gutschner, T.; Hämmerle, M.; Eißmann, M.; Hsu, J.; Kim, Y.; Hung, G.; Revenko, A.; Arun, G.; Stentrup, M.; Groß, M.; et al. The noncoding RNA MALAT1 is a critical regulator of the metastasis phenotype of lung cancer cells. Cancer Res. 2013, 73, 1180-1189, doi:10.1158/0008-5472.CAN-12-2850.

35. Jia, P.; Wu, N.; Jia, D.; Sun, Y. Downregulation of MALAT1 alleviates saturated fatty acid-induced myocardial inflammatory injury via the miR-26a/HMGB1/TLR4/NF-kB axis. Diabetes, Metab. Syndr. Obes. Targets Ther. 2019, 12, 655-665, doi:10.2147/DMSO.S203151.

36. Yan, C.; Chen, J.; Chen, N. Long noncoding RNA MALAT1 promotes hepatic steatosis and insulin resistance by increasing nuclear SREBP-1c protein stability. Sci. Rep. 2016, 6, 1-11, doi:10.1038/srep22640.

37. Liu, L.; Tan, L.; Yao, J.; Yang, L. Long non-coding RNA MALAT1 regulates cholesterol accumulation in ox-LDL-induced macrophages via the microRNA-17-5p/ABCA1 axis. Mol. Med. Rep. 2020, 21, 1761-1770, doi:10.3892/mmr.2020.10987.

38. Ebrahimi, R.; Toolabi, K.; Jannat Ali Pour, N.; Mohassel Azadi, S.; Bahiraee, A.; Zamani-Garmsiri, F.; Emamgholipour, S. Adipose tissue gene expression of long non-coding RNAs; MALAT1, TUG1 in obesity: Is it associated with metabolic profile and lipid homeostasis-related genes expression? Diabetol. Metab. Syndr. 2020, 12, 36, doi:10.1186/s13098-020-00544-0.

39. Carter, S.; Miard, S.; Boivin, L.; Sallé-Lefort, S.; Picard, F. Loss of Malat1 does not modify age- or diet-induced adipose tissue accretion and insulin resistance in mice. PLoS One 2018, 13, doi:10.1371/journal.pone.0196603.

40. Xu, Y.; Du, J.; Zhang, P.; Zhao, X.; Li, Q.; Jiang, A.; Jiang, D.; Tang, G.; Jiang, Y.; Wang, J.; et al. MicroRNA-125a-5p Mediates 3T3-L1 Preadipocyte Proliferation and Differentiation. Molecules 2018, 23, 317, doi:10.3390/molecules23020317.

41. Cheng, L.; Nan, C.; Kang, L.; Zhang, N.; Liu, S.; Chen, H.; Hong, C.; Chen, Y.; Liang, Z.; Liu, X. Whole blood transcriptomic investigation identifies long non-coding RNAs as regulators in sepsis. J. Transl. Med. 2020, 18, 217, doi:10.1186/s12967-020-02372-2.

42. Takahashi, K.; Sakurai, N.; Emura, N.; Hashizume, T.; Sawai, K. Effects of downregulating GLIS1 transcript on preimplantation development and gene expression of bovine embryos. J. Reprod. Dev. 2015, 61, 369-374, doi:10.1262/jrd.2015-029.

43. Scoville, D.W.; Kang, H.S.; Jetten, A.M. GLIS1-3: Emerging roles in reprogramming, stem and progenitor cell differentiation and maintenance. Stem Cell Investig. 2017, 4, doi:10.21037/sci.2017.09.01.

44. Deming, Y.; Li, Z.; Kapoor, M.; Harari, O.; Del-Aguila, J.L.; Black, K.; Carrell, D.; Cai, Y.; Fernandez, M.V.; Budde, J.; et al. Genome-wide association study identifies four novel loci associated with Alzheimer's endophenotypes and disease modifiers. Acta Neuropathol. 2017, 133, 839-856, doi:10.1007/s00401-017-1685-y.

45. Song, W.; Chen, Y.P.; Huang, R.; Chen, K.; Pan, P.L.; Li, J.; Yang, Y.; Shang, H.F. GLIS1 rs797906: An increased risk factor for late-onset Parkinson's disease in the han Chinese population. Eur. Neurol. 2012, 68, 89-92, doi:10.1159/000337955.

46. Tosic, M.; Allen, A.; Willmann, D.; Lepper, C.; Kim, J.; Duteil, D.; Schüle, R. Lsd1 regulates skeletal muscle regeneration and directs the fate of satellite cells. Nat. Commun. 2018, 9, doi:10.1038/s41467-017-02740-5.

47. Hou, L.; Shi, J.; Cao, L.; Xu, G.; Hu, C.; Wang, C. Pig has no uncoupling protein 1. Biochem. Biophys. Res. Commun. 2017, 487, 795-800, doi:10.1016/j.bbrc.2017.04.118.

48. Joshi, H.; Vastrad, B.M.; Joshi, N. Distinct Molecular Mechanisms Analysis of Obesity Based on Gene Expression Proles. Res. Sq. 2020, doi:10.21203/rs.3.rs-95029/v1.

49. Chessler, S.D.; Fujimoto, W.Y.; Shofer, J.B.; Boyko, E.J.; Weigle, D.S. Increased plasma leptin levels are associated with fat accumulation in Japanese Americans. Diabetes 1998, 47, 239-243, doi:10.2337/diab.47.2.239.

50. Foote, A.P.; Hales, K.E.; Kuehn, L.A.; Keisler, D.H.; King, D.A.; Shackelford, S.D.; Wheeler, T.L.; Freetly, H.C. Relationship of leptin concentrations with feed intake, growth, and efficiency in finishing beef steers. J. Anim. Sci. 2015, 93, 4401-4407, doi:10.2527/jas.2015-9339.

51. Perdomo, G.; Kim, D.H.; Zhang, T.; Qu, S.; Thomas, E.A.; Toledo, F.G.S.; Slusher, S.; Fan, Y.; Kelley, D.E.; Dong, H.H. A role of apolipoprotein D in triglyceride metabolism. J. Lipid Res. 2010, 51, 1298-1311, doi:10.1194/jlr.M001206.

52. Chen, H.; Mo, D.; Li, M.; Zhang, Y.; Chen, L.; Zhang, X.; Li, M.; Zhou, X.; Chen, Y. MiR-709 inhibits 3T3-L1 cell differentiation by targeting GSK3 $\beta$ of Wnt/ $\beta$-catenin signaling. Cell. Signal. 2014, 26, 2583-2589, doi:10.1016/j.cellsig.2014.07.017. 
53. Johnson, R.; Guigó, R. The RIDL hypothesis: Transposable elements as functional domains of long noncoding RNAs. RNA 2014, 20, 959-976, doi:10.1261/rna.044560.114.

54. Sindhu, S.; Akhter, N.; Kochumon, S.; Thomas, R.; Wilson, A.; Shenouda, S.; Tuomilehto, J.; Ahmad, R. Increased Expression of the Innate Immune Receptor TLR10 in Obesity and Type-2 Diabetes: Association with ROS-Mediated Oxidative Stress. Cell. Physiol. Biochem. 2018, 45, 572-590, doi:10.1159/000487034.

55. Goyenechea, E.; Crujeiras, A.B.; Abete, I.; Martínez, J.A. Expression of two inflammation-related genes (RIPK3 and RNF216) in mononuclear cells is associated with weight-loss regain in obese subjects. J. Nutrigenet. Nutrigenomics 2009, 2, 78-84, doi:10.1159/000210452.

56. Skorobogatko, Y.; Dragan, M.; Cordon, C.; Reilly, S.M.; Hung, C.W.; Xia, W.; Zhao, P.; Wallace, M.; Lackey, D.E.; Chen, X.W.; et al. RalA controls glucose homeostasis by regulating glucose uptake in brown fat. Proc. Natl. Acad. Sci. U. S. A. 2018, 115, 7819-7824, doi:10.1073/pnas.1801050115.

57. Singh, U.P.; Singh, N.P.; Murphy, E.A.; Singh, S.K.; Price, R.L.; Nagarkatti, M.; Nagarkatti, P.S. Adipose T cell microRNAs influence the T cell expansion, microbiome and macrophage function during obesity. J. Immunol. $2018,200$.

58. Xia, S.F.; Duan, X.M.; Cheng, X.R.; Chen, L.M.; Kang, Y.J.; Wang, P.; Tang, X.; Shi, Y.H.; Le, G.W. Role of miR-383 and miR-146b in different propensities to obesity in male mice. J. Endocrinol. 2017, 234, 201-216, doi:10.1530/JOE-17-0044. 